\title{
A Theoretical Model of the Water Vapor Diffusion through the Surface of Cloud Droplets
}

\author{
Raúl César Pérez
}

Lihando, Ceds. Department of Subjects Basics, National Technological University, Regional Mendoza Faculty, Rodriguez 257. Mendoza, Argentina

\begin{abstract}
In order to understand better the dynamics of formation, growth and evaporation process of the cloud drops, it had developed a mathematical and physics model, using the equation of the vapor diffusion law get cross its surface, taken into consideration that it has a spherical form. When the spherical drop is formed, its time of live will depend on its radio value $a$; so, on the one hand, if the $a$ value is bigger than critical radio value, then the drop growth because the condensation process win to the evaporation process, on the other hand, if the $a$ value is less than critical radio value, then the cloud drop will be evaporated. To the case the drop growth, the condensation process to the vapor diffusion gets cross its surface government the phenomenal dynamical. This important process is theoretically modeling in order to get a bigger understanding to design more efficient method of modifying them in a benefit way.
\end{abstract}

Key words: Drop, diffusion, model, water vapor.

\section{Introduction}

When a cloud drop growth to condensation process, its size changes to the water vapor molecule diffusion law, and its equation is [1]:

$$
\frac{\partial \rho_{v}}{\partial t}=D_{v} \nabla^{2} \rho_{v}
$$

The right side of the Eq. (1) represents the density variation of water vapor to the water vapor molecule flux get cross of the drop surface; on the other hand, the left side represents the density variation of water vapor on the time.

The $D_{v}$ is the water vapor diffusion coefficient, and it depends on the temperature present, and it is given to the expression [2]:

$$
D_{v}(T)=\frac{0.211}{P}\left(\frac{T}{273}\right)^{1.94}\left[\frac{m^{2}}{S}\right]
$$

where, $T$ is the temperature (Kelvin scale), and $p$ is the atmospheric pressure $(\mathrm{Pa})$.

If the cloud drop is considerate like as spherical and isotropic body with a radio $a$, then the water vapor

Corresponding author: Raúl César Pérez, Ph.D., research fields: earth, sea and atmospherics science. molecule flux of the Eq. (1) depends only on the radial distance $r$, and does not depend the angular variables; so the Eq. (2) is possible to put on spherical coordinate as:

$$
\frac{\partial \rho_{v}}{\partial t}=D_{v}(T) \frac{1}{r^{2}} \frac{\partial}{\partial r}\left[r^{2} \frac{\partial \rho_{v}}{\partial r}\right]
$$

In order to resolve the Eq. (3), one boundary condition that is possible to use is that at infinite radial distance $r$, the water vapor density value is approximate equal to the free atmospheric air density $\rho_{v}(\infty)[3]:$

$$
\begin{aligned}
& \rho_{v}(\infty)=\left(\frac{p_{0}}{R T}\right) e^{-\left(\frac{g z}{R T}\right)}= \\
& =\left(\frac{352.85}{T}\right) e^{-\left(\frac{0.034 z}{T}\right)}\left[\frac{K g}{m^{3}}\right]
\end{aligned}
$$

where, $p_{0}=101,235 \mathrm{~Pa}$ is the atmospheric pressure on the ground, $g=9.8 \mathrm{~m} / \mathrm{S}^{2}$ is the gravity acceleration value and $R=286.9 \mathrm{JKg}^{-1} \mathrm{~K}^{-1}$ is the universal gases constant. The $z$ parameter is the atmosphere height in meters.

How it can see, the boundary conditions (4) depend on the temperature value $T$ and the height $z$. 


\section{Material and Methods}

In order to resolve the differential Eq. (3), the separable variable method is used; so, it is possible to propose a solution function of the type:

$$
\rho_{v}(r, t)=F(r) . T(t)
$$

where, $F(r)$ is a function that depends only on the radial distance $r$, and $T(t)$ is a function that depends only on the time. Introducing Eq. (5) in the Eq. (3) and operating them, we obtain:

$$
D(T) \frac{1}{T} \frac{d T}{d t}=\frac{1}{F} \frac{1}{r^{2}} \frac{d}{d r}\left[r^{2} \frac{d F}{d r}\right]=-k^{2}
$$

The function solution of the left side, depends only on the time; on other hand, the central part has the function solution that only depends on the radial coordinate $r$. Then, both are equal to each other only if both are equal at the constant value that is called on the right side as $-k^{2}$.

So, it can resolve the differential Eq. (3) like a separate equations system:

$$
\begin{gathered}
\frac{1}{F} \frac{1}{r^{2}} \frac{d}{d r}\left[r^{2} \frac{d F}{d r}\right]=-k^{2} \\
D(T) \frac{1}{T} \frac{d T}{d t}=-k^{2}
\end{gathered}
$$

In order to resolve Eq. (7a), it can propose as solution function of trial:

$$
F(r)=\frac{e^{\alpha r}}{r}
$$

So, introducing Eq. (8) in Eq. (7a) and operating it, the next result is obtained:

$$
F(r)=\frac{A e^{k r}+B e^{-k r}}{r}
$$

To obtain the complete solution, it is necessary to find the function $T(t)$ that satisfies the differential Eq. (7b):

$$
\frac{d T}{T}=\frac{-k^{2}}{D_{v}} d t
$$

If both members of the Eq. (10) are integrated, the solution function obtained is:

$$
T(t)=T_{0} e^{\frac{-k^{2}}{D_{v}} t}
$$

where, $T_{0}=T(0)$ is the initial value of the solution function, when the diffusion process begins $(t=0)$. If this result and Eq. (9) are introduced in Eq. (5), it is obtained:

$$
\rho(r, t)=\frac{\left[A e^{k r}+B e^{-k r}\right]}{r} \cdot T_{0} e^{\frac{-k^{2}}{D v} t}
$$

Into the cloud drop that has a mass $M$, the water vapor molecule flux produces change in its mass $M$ on the time given to [1]:

$$
\dot{M}_{d}=4 \pi a^{2} D_{v}(T) \frac{d \rho_{v}}{d t}
$$

Deriving respect to the time Eq. (12), and introducing the result obtained to Eq. (13), it is arrived to:

$$
\dot{M}_{d}=-4 \pi a^{2} k^{2} T_{0} \frac{\left[A e^{k r}+B e^{-k r}\right]}{r} e^{\frac{-k^{2}}{D_{v}} t}
$$

When the radial distance $r$ tends to infinity, the water vapor density value must tend to constant value to free atmospheric air $\rho_{v}(\infty)$; then its mass variation on the time must be zero $\left(\dot{M}_{d}=0\right)$. Appling this boundary condition to Eq. (14), and using the L'Hopital rule in order to remove the discontinuity, it is possibly obtained:

$$
\dot{M}_{d}=-4 \pi a^{2} k^{2} \cdot T_{0}\left(A e^{\infty}-B e^{-\infty}\right)=0
$$

This expression is true only if the mass variation on the time value does not diverge. Then it is necessary that the constant value $A$ is zero $(A=0)$. With this result, Eq. (14) is:

$$
\dot{M}_{d}=C 4 \pi a^{2} k^{2} \frac{e^{-k r}}{r} e^{\frac{-k^{2}}{D_{v}} t}
$$

Where $C$ is a constant value that results of the product $C=B \cdot T_{0}$.

The interest of this work is to know how is the drop mass variation on the time, to effect of the water vapor diffusion get cross its surface with radio $a$.

If the mentioned study is made on this way, Eq. (16) is:

$$
\dot{M}_{d}=C .4 \pi a k e^{-k a} e^{\frac{-k^{2}}{D_{v}} t}
$$

Now, in order to determinate the $C$ value, it is necessary to find other relationship that together Eq. (17) forms an equations system. 
It is the common sense to think that the atmospheric air close to drop is saturated, especially on the drop surface. The change de drop mass on the time in this situation is given to next equation [1]:

$$
\dot{M}_{d}=\frac{4 \pi a}{Q_{c}+Q_{d}}\left[S-\frac{\alpha}{a}+\frac{\beta}{a^{3}}\right]
$$

where, $S$ is the saturation value, $Q_{c}$ is the interchange heat to conduction, $Q_{d}$ is the interchange heat to diffusion and its expressions are:

$$
S \equiv \frac{e(\infty)}{e_{S}(\infty)}-1=\frac{h}{100}-1
$$

In the saturation expression $S, e(\infty)$ is the water vapor pressure, $e_{s}(\infty)$ is the water vapor pressure, and $h$ is the relativity moist present.

$$
Q_{c}=\frac{L^{2}}{\kappa R_{v} T^{2}}=\frac{11049.9978}{4.39 T+0.071 T^{3}} 10^{6}[\text { joules }]
$$

In Eq. (20), $L$ represents the evaporation latent heat value $\left(L=2257.10^{3} \mathrm{Joule} / \mathrm{Kg}\right) . R_{v}$ is the water vapor gases constant, whose value is equal to 461 (Joule. $\mathrm{Kg}^{-1} \cdot \mathrm{K}^{-1}$ ); and $\kappa$ is the atmospheric air thermal conductivity, whose value depends on the temperature to the expression [2]:

$$
\kappa=(4.39+0.071 T)\left[\frac{\mathrm{Joule}}{\mathrm{m}^{2}}\right]
$$

The interchange heat to diffusion $Q_{d}$ expression is

$Q_{d}=\frac{R_{v} T}{D_{v} e_{s}(\infty)}=116430738 \frac{p}{e_{s}(\infty)} T^{-0.94}[$ joules $](22)$

Into Eq. (22) had been replaced in the Eq. (2).

If Eq. (18) is equal to Eq. (17), evaluating them at the initial time $(t=0)$, and operating, it is possible to obtain the expression to $C$ constant:

$$
C=\frac{S-\frac{\alpha}{a}+\frac{\beta}{a^{3}}}{k e^{-k a}\left(Q_{c}+Q_{d}\right)}
$$

\section{Results}

Then, if it is introducing Eq. (23) into Eq. (17), result is:

$$
\dot{M}_{d}=4 \pi a \frac{\frac{h}{100}-1-\frac{\alpha}{a}+\frac{\beta}{a^{3}}}{\left(Q_{c}+Q_{d}\right)} e^{\frac{-k^{2}}{D_{v}} t}
$$

The $\alpha$ coefficient, represents the surface curvature influence in the diffusion process, and its value depends on the temperature and is equal to:

$$
\propto=\frac{2 \sigma_{v l}}{\rho_{l} R_{v} T}=\left(-0.759+538.234 T^{-1}\right) 10^{-9} \mathrm{~m}
$$

In Eq. (25), $\sigma_{v l}$ is the drop surface stress, and its value also depends on the temperature to expression [4]:

$$
\sigma_{l v}=(-0.175 T+124.063) 10^{-3} \frac{\text { joule }}{m}
$$

This result was used into Eq. (25).

The coefficient represents the effect of the salt particles existence into the drop. The equation to calculate it is:

$$
\beta=\frac{3 i_{v h} m_{s} M_{w}}{4 \pi \rho_{l} M_{s}}=0.00429718 \frac{i_{v h} m_{s}}{M_{S}}\left[\mathrm{~m}^{3}\right]
$$

In the above equation, $i_{v h}$ is the Van't Hoff's factor, and it is equal at the ions number of the salt molecule separate; $m_{s}$ is the solute mass present into the drop and $M_{S}$ is molecular weight.

In order to use Eq. (22) to calculate the interchange heat to diffusion $Q_{d}$, it is necessary to know the saturation water vapor pressure, whose value depends on the temperature $T$, in agree with the Clasius-Clapeyron's law [5]:

$$
e_{S}(T)=e_{S}\left(T_{0}\right) e^{-\frac{\Delta H}{R_{v}}\left(\frac{1}{T}-\frac{1}{T_{0}}\right)}
$$

$H$ is the water vapor enthalpy, and its approximate value is $2257 \times 10^{3}$ [joule $/ \mathrm{kg}$ ].

So, in order to calculate the saturation water vapor pressure $e_{S}(T)$ using Eq. (24) to a determinate temperature $T$, if known the saturation water vapor pressure value $e_{S}\left(T_{0}\right)$, at the temperature $T_{0}$, for example:

$$
e_{s}(273.16 K)=999.84\left[\frac{K g}{\mathrm{~m}^{2}}\right]
$$

Using the data in Eq. (26), it is possible to write it like this:

$$
e_{S}(T)=999.84 e^{-4895.8785\left(\frac{1}{T}-0.00366\right)}\left[\frac{\mathrm{Kg}}{\mathrm{m}^{2}}\right]
$$

With the results obtained in the equations from (19) to (27), and the expression (2) in Eq. (24), it is possible to model the drop mass variation on the time 
by diffusion of water vapor to get cross its surface.

But, before using the model, the $k$ parameter must be measured to by experimental methods.

\section{Discussion and Acknowledgments}

From the results obtained, and if Eq. (24) is observed, it is possible to describe the drop mass variation on the time to vapor water diffusion effect get cross it surface; and get out an important conclusion:

(1) In order to use the model, it is necessary to measure in the laboratory in indirect way the $k$ parameter, the procedure may be to measure the drop mass at different time and with its value, to calculate the $k$ value.

(2) The temporal variation of the drop mass, depends on the diffusion coefficient, and the temperature, pressure and humidity of the present atmospheric air.
(3) The spherical cloud drop radio $a$ is a parameter that has an important influence on the mass change.

(4) The temporal behavior has an exponential decay at zero. Its time of life depends of parameter $k$ and the water vapor diffusion $D_{v}$.

(5) In order to use this model, it is necessary to get any data by direct measurement.

\section{References}

[1] Houze, Jr., and Robert, A. 1973. Clouds Dynamics. Academic. Press.

[2] Seinfeld, J. H., and Pandis, S. N. 1997. Atmospheric Chemistry and Physics from Air Pollution to Climate Change. USA: WILEY Interscience.

[3] www.google.com.ar/\#q=valor + de + la + densidad + media $+d$ el+aire+atmosferico.

[4] Pérez, Raúl C. 2012. "Study of the Particle Size Influence in the Cloud Seeding in Order to Get in Efficient Operations in the Weather Modification." Journal of Environmental Science and Engineering (JESE). 12 USA.

[5] http://es.wikipedia.org/wiki/ecuac\% $\% 3 \%$ n. 